\title{
Acupuntura e analgesia: aplicações clínicas e principais acupontos
}

\author{
Acupuncture and analgesia: clinical applications and main acupoints
}

\author{
Marilda Onghero Taffarel ${ }^{\mathrm{I}}$ Patricia Maria Coletto Freitas ${ }^{\mathrm{II}}$
}

\section{- REVISÃO BIBLIOGRÁFICA -}

\section{RESUMO}

A dor é uma resposta protetora do organismo a estímulos nocivos, que resulta em efeitos indesejáveis quando não controlada. A analgesia pode ser promovida mediante a utilização de vários tipos de fármacos. No entanto, estes podem causar efeitos adversos de acordo com a espécie e condição física do paciente. A acupuntura tem se mostrado eficaz como coanalgésico pela capacidade de diminuir a quantidade de fármacos utilizados para o controle da dor e raramente ser contraindicada. Objetivou-se com este trabalho fazer uma breve revisão sobre as aplicações clínicas e os efeitos fisiológicos da acupuntura nos mecanismos da dor, bem como demonstrar os principais pontos de acupuntura utilizados para analgesia em animais. A pesquisa foi realizada em bases de dados eletrônicas por palavra-chave, durante o período de março a dezembro de 2008.

Palavras-chave: medicina tradicional chinesa, acupontos, animais.

\section{ABSTRACT}

Pain is a protective response of the body to harmful stimulus, which results in undesirable effects if not controlled. Analgesia can be achieved with the use of different types of drugs. However, these drugs can cause adverse effects according to species and patient physical condition. Acupuncture has been proved to be an effective analgesic adjuvant, by the capacity to decrease the amount of drug used for pain control, rarely contra-indicated. The aim of this paper was to review the physiological effects of acupuncture on pain mechanisms, and demonstrate the main acupoints used for animal analgesia. The search was done in electronic search database using key words, in 2008.

Key words: traditional chinese medicine, acupoints, animals.

\section{INTRODUÇÃO}

AAssociação Internacional para o Controle da Dor (IASP) define dor como "uma experiência emocional e sensorial desagradável, associada a lesões reais ou potenciais e descrita em termos de tais lesões" (MENEZES, 2004). Perante isso, fármacos são administrados com objetivo de controlar a dor em animais, como opióides, anti-inflamatórios não esteroidais, anestésicos locais, agonistas $\alpha-2$ e anestésicos dissociativos como a cetamina (LASCELLES \& WATERMAN, 1997; FANTONI \& MASTROCINQUE, 2002). Entretanto, apesar dos avanços da Medicina, protocolos para o controle da dor ainda requerem estudos, especialmente em pacientes pediátricos, geriátricos, oncológicos e diabéticos, nos quais os fármacos utilizados podem promover sedação, depressão respiratória e distúrbios gastrointestinais (FANTONI \& MASTROCINQUE, 2002; PARRIS \& SMITH, 2003). Dessa forma, métodos complementares, como a acupuntura, estão sendo cada vez mais utilizados para o tratamento da dor (PARRIS \& SMITH, 2003).

O tratamento e controle da dor têm grande importância devido aos efeitos deletérios promovidos pelo desencadeamento fisiológico do processo doloroso, não devendo ser negligenciado. A utilização da analgesia profilática e terapia analgésica multimodal

'Laboratório de Cirurgia Experimental e Terapia Intensiva, Universidade Federal do Espírito Santo (UFES), Alegre, ES, Brasil. "Departamento de Medicina Veterinária, UFES. Rua Alto Universitário, s/n, CP 16, 29500-000, Alegre, ES, Brasil. E-mail: pcoletto@yahoo.com.br. Autor para correspondência. 
tem se mostrado eficaz, evitando a hiperalgesia e alodinia. Nesse sentindo, a acupuntura desponta como uma técnica promissora na área.

A acupuntura é uma técnica da Medicina Tradicional Chinesa (MTC) utilizada desde 2.000 a 3.000 anos antes de Cristo. No ocidente, a prática foi introduzida por missionários jesuítas há aproximadamente 300 anos. Porém, foi a partir de 1970 que esta passou a ser estudada, especialmente por seus efeitos analgésicos (PARRIS \& SMITH, 2003; SANTOS \& MARTELETE, 2004; AMMENDOLIA et al., 2008). Esta consiste na inserção de agulhas em pontos anatômicos específicos do corpo, com o objetivo de produzir efeito terapêutico ou analgésico (LIN, 2006). A MTC baseia-se nas teorias do Yin-Yang e dos Cinco Elementos (LUNA, 2002; LIN, 2006). Segundo seus conceitos, o campo eletromagnético da vida (Qi) no organismo flui por todos os órgãos, e a comunicação entre estes ocorre pelos meridianos. Alterações nesse fluxo manifestariam sintoma de acúmulo (Yang - quente, ativo) ou deficiência (Yin frio, passivo) de energia. A colocação de agulhas em pontos de Yin e Yang normaliza esse desequilíbrio (DRAEHMPAEL \& ZOHMANN, 1997; ULETT et al., 1998; LIN, 2006; POVOLNY, 2008).

Na Medicina Veterinária, a acupuntura é indicada no tratamento e controle da dor (LUNA, 2002), de traumas vertebrais, de doenças do disco intervertebral (HAYASHI et al., 2007) e da Síndrome de Horner idiopática (CHO \& KIM, 2008), dentre outros. Apesar de não abolir completamente a dor, produzindo hipoalgesia, a experiência clínica mostra que a acupuntura pode ser utilizada como única ferramenta no controle da dor em alterações musculoesqueléticas, mas não no pós-operatório (LUNA, 2002; XIE \& PREAST, 2007).

Dessa forma, objetivou-se com este estudo revisar os efeitos fisiológicos da acupuntura sobre os mecanismos da dor, assim como demonstrar os principais acupontos utilizados para analgesia em animais. Para esta revisão, foram realizadas buscas em bases de dados eletrônicos, tais como Scielo, Science Direct e PubMed, por palavra-chave, durante o período de março a dezembro de 2008.

Bases anatômicas e fisiológicas da acupuntura

$\mathrm{O}$ acuponto é definido como um ponto da pele de sensibilidade espontânea ao estímulo e à resistência elétrica reduzida. Possui um diâmetro de 0,1 a $5 \mathrm{~cm}$, entretanto é uma área de condutividade elétrica amplamente aumentada comparada às áreas da pele ao redor (SCHWARTZ, 2008). Estes estão localizados próximos a articulações e bainhas tendíneas, vasos, nervos e septos intramusculares, na ligação músculotendínea, nos locais de maior diâmetro do músculo e nas regiões de penetração dos feixes nervosos da pele (DRAEHMPAHEL \& ZOHMANN, 1997). Quando um ponto de acupuntura é puncionado, ocorre sensação de parestesia elétrica ou calor. Essa sensação é denominada como De Qi. Os animais demonstram essa sensação com um leve repuxo de pele, uma discreta sonolência ou uma breve tremor de cauda (HWANG \& EGERBACHER, 2006).

A acupuntura atua sobre o controle da dor por ativação de vias opióides e não opióides. De acordo com SANTOS \& MARTELETE (2004), a estimulação promovida por essa técnica ativa o sistema modulador da dor por hiperestimulação das terminações nervosas de fibras mielínicas A- $\delta$, responsáveis pela condução do estímulo aos centros medulares, encefálicos e eixo hipotálamo-hipofisário. Na medula espinhal, a modulação dos estímulos nociceptivos se dá por inibição pré-sináptica, devido à liberação de encefalinas e dinorfinas. No mesencéfalo, as encefalinas e a ativação do sistema central de modulação da dor resultam na liberação de serotonina e norepinefrina nos sistemas descendentes. KOO et al. (2008), ao estudarem os efeitos analgésicos da eletroacupuntura em ratos submetidos à torção do tarso, concluíram que a eletroacupuntura ativa neurônios bulboespinhais, o que resulta na liberação de noradrenalina e ativação de $\alpha 2$-adrenoreceptores do corno dorsal da medula, confirmando uma via não opioide de ação da eletroacupuntura.

Segundo DRAEHMPAHEL \& ZOHMANN (1997) e SANTOS \& MARTELETE (2004), a acupuntura estimula ainda o eixo hipotálamo-hipofisário a liberar ß-endorfinas na circulação sistêmica e no líquor. Paralelamente, ocorre liberação de hormônio adrenocorticotrófico, induzindo a liberação de cortisol. Entretanto, existem controvérsias acerca da ação dos hormônios corticóides no efeito anti-inflamatório da acupuntura (SCOGNAMILLO-SZABÓ, et al., 2004; LI et al., 2007). Além disso, YANG et al. (2008) relataram que o núcleo hipotalâmico supraóptico possui um importante papel na analgesia promovida pela acupuntura, pois secreta arginina-vasopressina e ocitocina, que promovem aumento no limiar da dor. A eletroacupuntura também modula a atividade dos receptores NMDA, que estão envolvidos no desenvolvimento da dor crônica (WANG et al., 2006).

Segundo ULETT et al. (1998), a analgesia promovida pela acupuntura também está relacionada com vias opioides, promovidas ao estimularem pontos periféricos do corpo. ZHANG et al. (2005) relataram que o efeito antiálgico da eletroacupuntura é mediado 
por receptores $\mu$, resultando na liberação de endomorfina/endorfina e receptores $\delta$ e liberando encefalina (ZHANG et al., 2004). Porém, LUNA (2002) descreveu que é necessário estímulo contínuo dos acupontos por 30 minutos para que ocorra essa liberação endógena de opioides.

Métodos de acupuntura

O ponto de acupuntura pode ser estimulado por acupressão, moxabustão, laserpuntura, aquapuntura e eletroacupuntura, dentre outras técnicas (LUNA, 2002; LIN, 2006). A acupressão consiste na aplicação de pressão sobre a superfície do corpo de uma forma geral (massagem) ou em pontos específicos. Normalmente não é utilizada por médicos veterinários, mas os proprietários podem ser treinados para utilizar essa técnica como forma de complementar a terapia com agulhas (ALTMAN, 2006). A moxabustão é o aquecimento do ponto de acupuntura com a queima de bastões de uma planta chamada Artemisia vulgaris. A técnica pode ser realizada de forma direta, queimando os bastões diretamente sobre a pele, ou indireta (ALTMAN, 2006). Outro método não invasivo consiste na utilização de Laser (Light Amplification by Stimulated Emission of Radiation), com rendimento de 1 a $10 \mathrm{~mW} \mathrm{~cm}^{-2}$ (soft-laser), em acupontos analgésicos (DRAEHMPAHEL \& ZOHMANN, 1997). A aplicação do soft-laser aumenta a síntese de adenosina trifosfato (ATP) na célula, causando hiperpolarização e bloqueio de estímulos menores, diminuindo a transmissão de impulsos dolorosos. SHEN et al. (2008) verificaram que o estímulo do acuponto E35 por aplicação de laser em pacientes com osteoartrite no joelho resultou em redução da dor clínica em $49 \%$.

A injeção de produtos medicinais nos acupontos é chamada de aquapuntura. É especialmente indicada na auriculoterapia. Também pode ser empregada para tratar lombalgias, melhorando a excreção de toxinas metabólicas e substâncias álgicas pela vias linfáticas (DRAEHMPAHEL \& ZOHMANN, 1997). De outra forma, a eletroacupuntura consiste na utilização de estímulo elétrico pelas agulhas, emitido por meio de um aparelho de eletroestimulação. LIU et al. (2007) afirmaram que a estimulação elétrica de acupontos é efetiva no alívio da dor central e periférica. $\mathrm{O}$ efeito analgésico desse método foi descrito em cirurgias abdominais (LIN et al., 2002), reparo de hérnia inguinal (CHU et al., 2003), toracotomia (WONG et al., 2006), como método complementar para analgesia em pacientes com câncer (ZHANG et al., 2007), e lesões na articulação tíbio-társica (KOO et al., 2008).
A auriculoterapia é indicada nas doenças agudas e reversíveis, para analgesia e adjuvante nas doenças crônicas (DRAEHMPAHEL \& ZOHMANN, 1997). ALIMI et al. (2003) comprovaram a eficácia da auriculoterapia como tratamento auxiliar na dor crônica neuropática em pacientes oncológicos. Apesar dos resultados promissores, as evidências para o uso da auriculoacupuntura no controle da dor ainda são inconclusivas (USICHENKO, LEHMANN \& ERNST, 2008). Comumente não se realiza em animais o agulhamento da orelha, mas sim a cauterização dos pontos (DRAEHMPAHEL \& ZOHMANN, 1997).

\section{Aplicação clínica}

No ocidente, segundo SANTOS \& MARTELETE (2004), a acupuntura é utilizada como adjuvante no tratamento da dor, pois promove hipoalgesia, haja vista a diferença de resposta individual a essa técnica para a abolição da dor (PARRIS e SMITH, 2003). Entretanto, a combinação de diversas modalidades analgésicas da acupuntura possibilita a realização até de procedimentos cirúrgicos (SANTOS \& MARTELETE, 2004).

$\mathrm{Na}$ Medicina Veterinária, a eletroacupuntura tem sido utilizada com fins de obtenção de hipoalgesia, devido à dificuldade de estimulação manual da agulha nos pacientes. A intensidade da estimulação é regulada de acordo com o limite individual da dor do paciente, manifestado por estremecimentos rítmicos da pele e dos músculos adjacentes. A frequência e a tensão elétrica devem ser variadas, pois muitas vezes o paciente acostuma-se a essa estimulação. Além disso, o alcance da hipoalgesia é influenciado pela escolha dos acupontos e da forma de estimulação (LUNA, 2002).

Assim, a escolha dos pontos deve ocorrer de acordo com o percurso do meridiano e a distribuição dos nervos que atravessam a área de acontecimento da dor. Utiliza-se eletroacupuntura de alta frequência para analgesia de pontos do tronco e de baixa frequência para pontos dos membros (DRAEHMPAHEL \& ZOHMANN, 1997). LIN et al. (2002) testaram no homem estímulo de baixa e alta frequência em pacientes submetidos à cirurgia abdominal baixa e verificaram que ambas diminuem a necessidade de morfina no pósoperatório. No entanto, a estimulação de alta frequência foi mais eficaz para o controle da dor. De acordo com ULETT (1992), estímulo elétrico de baixa frequência (2 a $5 \mathrm{~Hz}$ ) induz a liberação central de endorfinas e encefalinas, resultando em analgesia em 10 a 20 minutos com efeito cumulativo. Entretanto, o estímulo de alta frequência $(100$ a $200 \mathrm{~Hz})$ promove liberação de serotonina, epinefrina e norepinefrina, resultando em analgesia não cumulativa. A frequência determina a 
classe de neuropeptídeos liberados no sistema nervoso central. De forma geral, é possível afirmar que baixas frequências (até $10 \mathrm{~Hz}$ ) liberam encefalina, betaendorfina e endomorfina, responsáveis pela analgesia de longa duração. Altas frequências $(100 \mathrm{~Hz})$ liberam dinorfina e produzem analgesia imediata (HAN, 2004). LUNA (2002) recomenda a utilização de corrente de baixa e alta frequência de forma sequencial e alternada para haver melhor resposta analgésica. CASSU et al. (2008) demonstraram que a eletroacupuntura bilateral produz analgesia com menor período de latência, maior intensidade e efeito mais duradouro que a estimulação unilateral.

\section{Principais pontos analgésicos}

Para a Medicina Tradicional Chinesa, a dor resulta de condição de excesso ou deficiência de Qi ou sangue. A estimulação e os pontos proximais, locais e distais restauram o equilíbrio e normalizam a causa da dor. Um dos princípios envolve escolher pontos proximais e distais à área afetada. Contudo, protocolos já estudados podem ser úteis para a melhor escolha dos acupontos (KLIDE \& GAYNOR, 2006).

$B I N A O$ (IG 14) localiza-se na tuberosidade deltóidea, onde se insere a parte acromial do músculo deltóide, a uma profundidade de $1 \mathrm{~cm}$ (Figura 1). Ele é indicado para dores na região do ombro e antebraço (DRAEHMPAHEL \& ZOHMANN, 1997).

QUCHI (IG 11) é um ponto de tonificação e sua localização se dá com o cotovelo levemente dobrado, no meio, entre o final da dobra do cotovelo e o epicôndilo lateral do úmero, na origem do músculo extensor carporradial e profundamente na inserção do tendão do músculo braquial, em uma profundidade de 1 a $2 \mathrm{~cm}$ (Figura 2). Ele é indicado para dores no ombro, cotovelo e antebraço, epicondilite, obstipações e paralisia de radial. Quando associado ao IG10 e IG4, é indicado para tratamento da dor na osteocondrite do membro torácico (DRAEHMPAHEL \& ZOHMANN, 1997; HWANG \& LIMEHOUSE, 2006).

HEGU (IG 4) localiza-se na extremidade medial do primeiro metacarpiano, no meio da dobra de

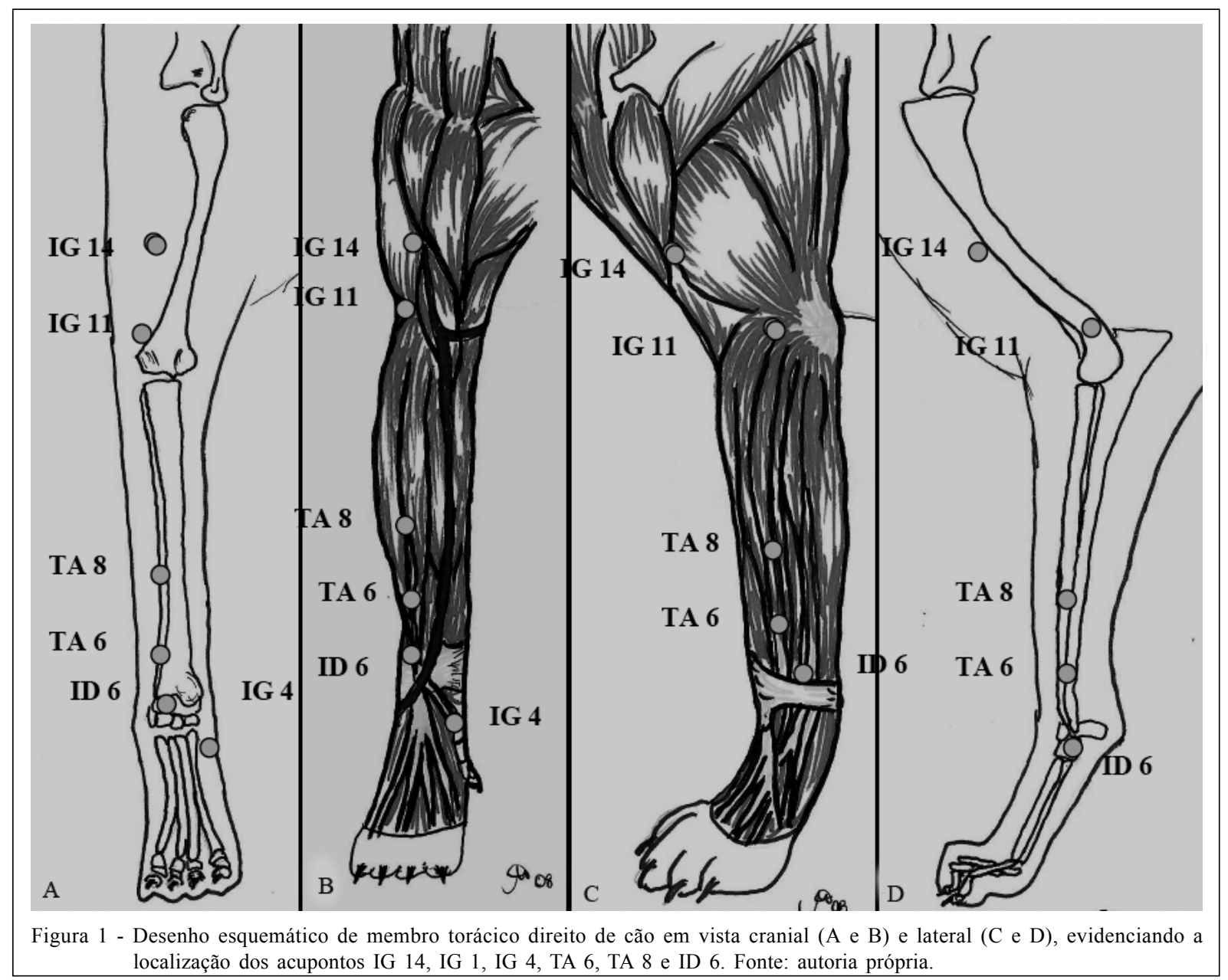

Ciência Rural, v.39, n.9, dez, 2009. 


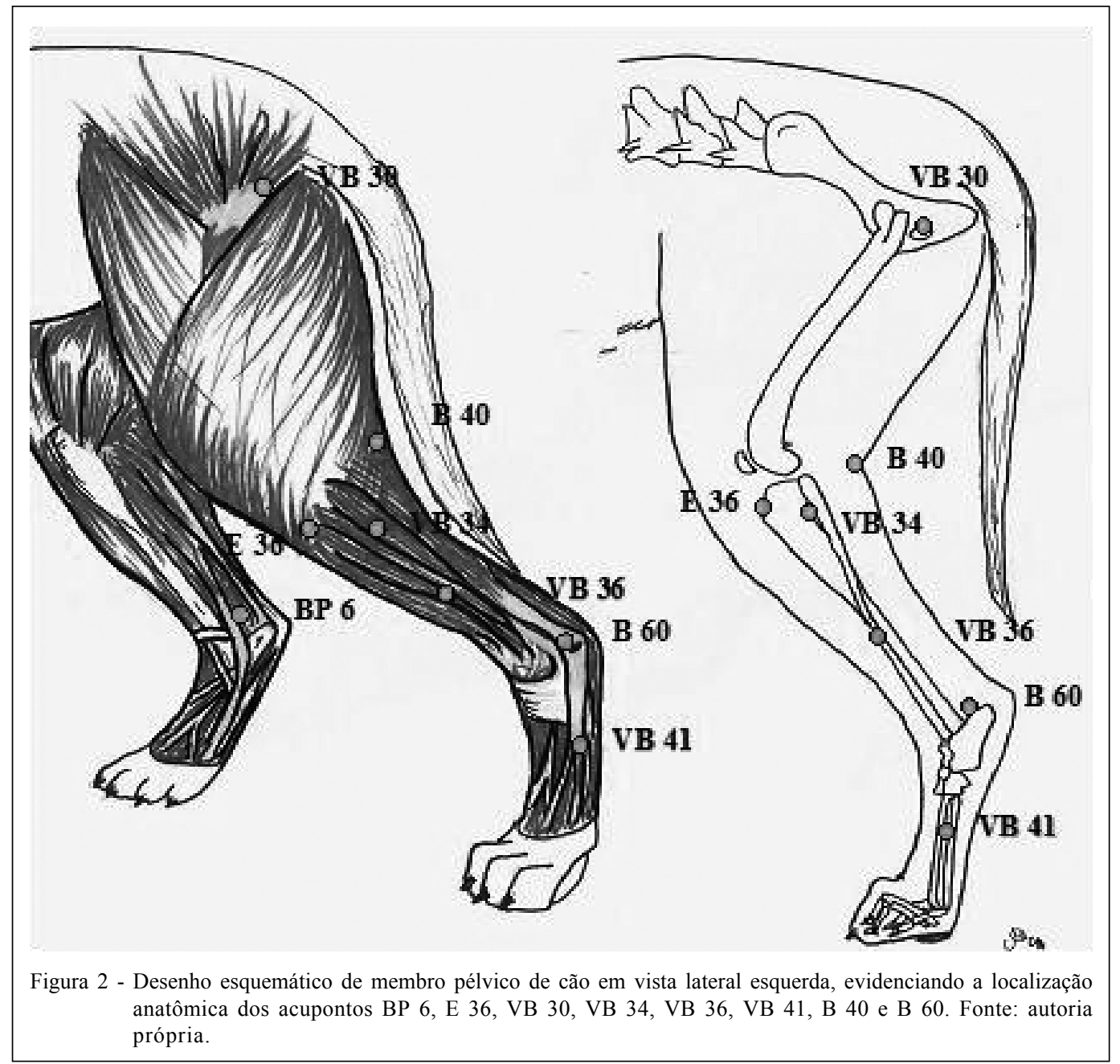

pele, após a abdução do primeiro metacarpiano. Ele é aplicado diagonal e distalmente a uma profundidade de 0,2 a $0,5 \mathrm{~cm}$ (Figura 1) e indicado para tratamento do membro anterior, de dores de dente e de mandíbula e dor funcional do olho. Além disso, ele é indicado em conjunto com IG 10 e IG 11 para tratamento de osteocondrite do membro anterior e como analgésico em conjunto com os pontos E 36 e BP 6 (DRAEHMPAHEL \& ZOHMANN, 1997). KOO et al. (2008) utilizaram esse ponto associado ao ID $6 \mathrm{com}$ sucesso para analgesia em ratos submetidos à torção da articulação tíbio-társica. Também ALMEIDA et al. (2008) verificaram que esse acuponto, quando associado ao BP 6 , reduz o consumo do anestésico isofluorano em gatas submetidas à ovariohisterectomia eletiva, indicando um efeito analgésico dessa associação.

SANYANGLUO (TA 8) localiza-se no quarto inferior do antebraço, cerca de 3 a 4 diâmetros de dedo, proximal à dobra dorsal do carpo em uma linha imaginária no meio desta dobra e do epicôndilo lateral do úmero, na passagem do músculo-tendão do músculo extensor digital comum e extensor digital lateral (Figura 1). $\mathrm{O}$ agulhamento se faz perpendicular à pele, a uma profundidade de 1 a $2 \mathrm{~cm}$. Ele é indicado para analgesia do membro torácico e associado ao TA 6 é indicado para hipoalgesia do tórax (DRAEHMPAHEL \& ZOHMANN, 1997).

YANGLAO (ID 6) localiza-se dorsalmente entre o osso carpo-ulnar e o processo estiloide lateral a uma profundidade de 0,5 a $1 \mathrm{~cm}$ (Figura 1). Ele é indicado para dores na articulação do carpo, edemas e tensões do pescoço e dores no tórax (DRAEHMPAHEL \& ZOHMANN, 1997; HWANG \& LIMEHOUSE, 2006). WEIZHONG (B 40) situa-se exatamente no meio da dobra da articulação fêmuro-tibio-patelar, entre o epicôndilo medial e lateral do fêmur (Figura 2). Sua profundidade varia de 1 a $6 \mathrm{~cm}$, de acordo com o tamanho do paciente. Ele é indicado em dores musculares e espasmos, dores da articulação fêmuro-tibio-patelar, 
dores abdominais e paraplegias traumáticas quando associado ao B 37, B 54, e B 60 (DRAEHMPAHEL \& ZOHMANN, 1997). UM et al. (2005) utilizaram B 40 associado ao VB 33, VB 34 e VC 8 com sucesso para tratamento de artrite crônica da articulação fêmuro-tíbiopatelar em cães.

KUNLUN (B 60) localiza-se entre a tuberosidade calcânea e o maléolo lateral, a uma profundidade de 2 a $5 \mathrm{~mm}$ (Figura 2). Ele é indicado em casos de torções e edema da articulação tíbio-tarsica, anquiloses, dores isquiáticas e síndrome cervical (DRAEHMPAHEL \& ZOHMANN, 1997), paralisia de membro pélvico e retenção de placenta (HWANG \& LIMEHOUSE, 2006). Quando associado ao B 37, B 40 e B 54, é utilizado em paraplegias traumáticas (DRAEHMPAHEL \& ZOHMANN, 1997).

HUANTIAO (VB 30) encontra-se caudodorsal ao trocânter maior, em aprofundamento atrás da articulação coxofemoral, a uma profundidade de 1 a $2 \mathrm{~cm}$ (Figura 2). Ele é indicado em doenças da articulação coxofemoral, dores e paralisias dos membros pélvicos e edemas (DRAEHMPAHEL \& ZOHMANN, 1997; HWANG \& LIMEHOUSE, 2006). ZHANG et al. (2007) verificaram que a eletroestimulação desse acuponto promoveu efeito analgésico em ratos com câncer ósseo metastático induzido. Além disso, WANG et al. (2006) demonstraram que o estímulo por eletroacupuntura dos pontos VB 30 e VB 34 foi capaz de modular a atividade dos receptores do tipo n-metild-aspartato (NMDA) em ratos.

YANGLINGQUAN (VB 34) está localizado em um aprofundamento ventro-caudal da cabeça fibular (Figura 2), na profundidade de $1 \mathrm{a} 4 \mathrm{~cm}$. Ele é indicado para edemas e dores na articulação do articulação fêmuro-tibio-patelar e membro pélvico (DRAEHMPAHEL \& ZOHMANN, 1997), paresia e paralisia de membro pélvico e doença de disco toracolombar (HWANG \& LIMEHOUSE, 2006). Segundo WONG et al. (2006), a eletroestimulação desse acuponto associado aos pontos VB 36 e TA 8 foi capaz de reduzir a dose necessária de morfina para analgesia pós-operatória de pacientes submetidos à toracotomia.

WAIQIU (VB 36) encontra-se no meio de uma linha imaginária entre o maléolo lateral e a cabeça fibular, diretamente na extremidade anterior da fíbula (Figura 2). Para localizar o WAIQIU, posiciona-se a agulha perpendicular à pele em uma profundidade de $1 \mathrm{a} 2 \mathrm{~cm}$. Ele é indicado especialmente em doenças agudas, em dores na nuca e na região hipocondríaca e na síndrome cervical. No homem promove analgesia nas cólicas da vesícula biliar(DRAEHMPAHEL \& ZOHMANN, 1997).

SANYINJIAO (BP 6) localiza-se atrás da extremidade medial da tíbia, na altura de uma linha vertical da tuberosidade calcânea (Figura 2), a uma profundidade de 1 a $2 \mathrm{~cm}$, de acordo com o tamanho do paciente, perpendicular à pele. Ele é indicado para dores abdominais, diarréia, ponto mestre em disfunções urogenitais, incontinência, dismenorréia (DRAEHMPAHEL \& ZOHMANN, 1997), analgesia para cirurgia abdominal, dentre outras indicações (HWANG \& LIMEHOUSE, 2006). JEONG \& NAM (2003) verificaram que o estímulo dos pontos BP 6, IG 4, E 36 e TA 8 diminuiu a concentração alveolar mínima do isofluorano em cães, indicando assim um possível efeito analgésico.

ZUSANLI (E 36) localiza-se em um aprofundamento lateral à tuberosidade tibial, na base do músculo tibial cranial (Figura 2), a uma profundidade de 0,5 a $2 \mathrm{~cm}$. Para analgesia, pode associá-lo ao BP 6 e IG 4 (DRAEHMPAHEL \& ZOHMANN, 1997). CASSU et al. (2008) associaram esse ponto ao VG 34 e verificaram sua eficácia para analgesia cutânea do tórax e abdome. CHU et al. (2003) utilizaram esse acuponto associado aos pontos BP 6, VB 27, VB 28, R 13, R 14 e ao agulhamento paraincisional para analgesia no transoperatório para reparo de hérnia inguinal no homem. Esses autores relataram que, em 33\% dos pacientes tratados, não foi necessário uso de outra técnica analgésica durante a cirurgia. LIN et al. (2002) observaram redução no consumo de morfina no pósoperatório de pacientes submetidos à cirurgia abdominal baixa tratados com eletroacupuntura nesse acuponto. PIGNATON et al. (2007) observaram que a eletroestimulação dos acupontos E 36 e VB 34 proporcionou redução no consumo de isofluorano e melhorou a estabilidade cardiorrespiratória, quando comparada com a morfina, durante ovariohisterectomia em gatas.

ZULINQI (VB 41) está localizado entre o quarto e o quinto metatarsiano, na extremidade lateral do tendão distal do músculo extensor digital longo, dorsolateral sobre a articulação tíbio-társica (Figura 2), a uma profundidade de $1 \mathrm{~cm}$ perpendicular à pele, ao lado do tendão (DRAEHMPAHEL \& ZOHMANN, 1997). Sua eletroestimulação, associado ao TA 5, promoveu diminuição do consumo de anestésico isofluorano e estabilidade cardiovascular em gatas submetidas à ovariohisterectomia (ZETUN et al., 2008).

\section{CONCLUSÕES}

A acupuntura mostra-se como uma alternativa de terapia adjuvante ao controle da dor, já que possui mínimos efeitos adversos e contraindicação. Ela pode ser utilizada para promover analgesia abdominal do membro torácico da coluna, do tórax e 
do membro pélvico. Apesar dos relatos e testes clínicos bem-sucedidos, a revisão da literatura científica demonstra a necessidade de realização de mais estudos científicos nas diferentes espécies animais para comprovar cientificamente a eficácia da acupuntura como método analgésico e melhorar a difusão da técnica na Medicina Veterinária.

\section{REFERÊNCIAS}

ALIMI, D. et al. Analgesic effect of auricular acupuncture for cancer pain: a randomized, blinded, controlled trial. Journal of Clinical Oncology, v.21, n.22, p.4120-4126, 2003. Disponível em $<$ http://www.jco.ascopubs.org $>$. Acesso em: 17 abr. 2008. doi: 10.1200/JCO.2003.09.011

ALMEIDA, S.D.S.R. et al. Eletroestimulação dos acupontos IG 4 e BP 6 sobre parâmetros cardiorrespiratórios e consumo do anestésico isofluorano em gatas submetidas à ovariosalpingohisterectomia eletiva. In: CONGRESSO BRASILEIRO DE CIRURGIA E ANESTESIOLOGIA VETERINÁRIA, 8., 2008, Recife, PB. Anais... Recife: CBCAV, 2008. CD Room.

ALTMAN, S. Técnicas e instrumentação. In: SHOEN, A.M. Acupuntura veterinária. Da arte antiga à medicina moderna. 2.ed. São Paulo: Roca, 2006. p.91-108.

AMMENDOLIA, C. et al. Evidence-informed management of chronic low back pain with needle acupuncture. Spine Journal, v.8, p.160-172, 2008 .

CASSU, R.N. et al. Electroacupuncture analgesia in dogs: is there a difference between uni-and bi-lateral stimulation? Veterinary Anaesthesia and Analgesia, v.35, p.52-61, 2008. Disponível em: < http://www3.interscience.wiley.com/cgibin/fulltext/119421052/PDFSTART>. Acesso em: 18 mar. 2008. doi:10.1111/j.1467-2995.2007.00347.x.

CHO, S.J.; KIM, O. Acupuncture treatment for idiopathic Horner's syndrome in a dog. Journal of Veterinary Science, v.9, n.1, p.117-119, 2008.

CHU, W.D. et al. Acupuncture anaesthesia in inguinal hernia repair. ANZ Journal of Surgery, v.73, p.125-127, 2003.

DRAEHMPAEL, D.; ZOHMANN, A. Acupuntura no cão e no gato. Princípios básicos e prática científica. São Paulo: Roca, 1997. 254p.

FANTONI, D.T.; MASTROCINQUE, S. Fisiopatologia e controle da dor. In: FANTONI, D.T.; CORTOPASSI, S.R.G. Anestesia em cães e gatos. São Paulo: Roca, 2002. p.323336.

HAN, J.S. Acupuncture and endorphins. Neuroscience Letters, v.361, n.1-3, p.258-261, 2004.

HAYASHI, A.M. et al. Electro-acupuncture and Chinese herbs for treatment of cervical intervertebral disk disease in a dog. Journal of Veterinary Science, v.8, n.1, p.95-98, 2007.

HWANG, Y.C.; LIMEHOUSE, J.B. Atlas de acupuntura canina. In: SHOEN, A.M. Acupuntura veterinária. Da arte antiga à medicina moderna. 2.ed. São Paulo: Roca, 2006. p.122146 .
HWANG, Y.C; EGERBACHER, M. Anatomia e classificação dos acupontos. In: SHOEN, A.M. Acupuntura veterinária. Da arte antiga à medicina moderna. 2.ed. São Paulo: Roca, 2006. p.17-23.

JEONG, S.M.; NAM, T.C. Effect of eletroacupuncture on minimum alveolar concentration of isofluorane in dogs. Journal of Veterinary Medical Science, v.65, n.1, p.145147, 2003.

KLIDE, A.M.; GAYNOR, J.S. Acupuntura para analgesia cirúrgica e pós-operatória. In: SHOEN, A.M. Acupuntura veterinária. Da arte antiga à medicina moderna. 2.ed. São Paulo: Roca, 2006. p.289-295.

KOO, S.T. et al. Electroacupuncture-induced analgesia in a rat model of ankle sprain pain is mediated by spinal alphaadrenoceptors. Pain, v.135, n.1-2, p.11-19, 2008.

LASCELLES, D.; WATERMAN, A. Analgesia in cats. In Practice, v.19, n.4, p.203-213, 1997.

LI A, et al. Corticosterone mediates electroacupunctureproduced anti-edema in a rat model of inflammation. BMC Complemententary and Alternative Medicine. v.14, n.7, p.27, 2007.

LIN, J.G. et al. The effect of high and low frequency electroacupuncture in pain after lower abdominal surgery. Pain, v.99, p.509-514, 2002.

LIN, Y.C. Perioperative usage of acupuncture. Pediatric Anesthesia, v.16, p.231-235, 2006.

LIU, H.X. et al. Repeated $100 \mathrm{~Hz}$ TENS for the treatment of chronic inflammatory hyperalgesia and suppression of spinal release of Substance P in monoarthritic rats. Evidence-Based Complementary and Alternative Medicine, v.4, p.65-75, 2007. Disponível em:< (http://creativecommons.org/licenses/ by-nc/2.0/uk/)>. Acesso em 14 abr. 2008. DOI: 10.1093/ecam/ ne1056.

LUNA, S.P.L. Emprego da acupuntura em anestesia. In: FANTONI, D.T.; CORTOPASSI, S.R.G. Anestesia em cães e gatos. São Paulo: Roca, 2002. p.337-343.

MENEZES, M.S. Anatomia e fisiopatologia da dor. In: MANICA, J. et al. Anestesiologia. Princípios e técnicas. 3.ed. Porto Alegre: Artmed, 2004. p.1251-1257.

PARRIS, W.C.V.; SMITH, H.S. Alternative pain medicine. Pain Practice, v.3, n.2, p.105-116, 2003.

PIGNATON, W. et al. Efeito da eletroacupuntura e da morfina sobre parâmetros cardiorrespiratórios e consumo de anestésico em gatas submetidas à ovariosalpingohisterectomia. Acta Scientiae Veterinarie, v.35, Supl.2, p.331-332, 2007.

POVOLNY, B. Acupuncture and traditional chinese medicine: an overview. Techniques in Regional Anesthesia and Pain Management, v.12, n.2, p.109-110, 2008.

SANTOS, L.M.M.; MARTELETE, M. Acupuntura no tratamento da dor. In: MANICA, J. et al. Anestesiologia. Princípios e técnicas. 3.ed. Porto Alegre: Artmed, 2004. p. 1307-1309. 
SCHWARTZ, C. Quatro patas cinco direções. Um guia de Medicina Chinesa para cães e gatos. São Paulo: Ícone, 2008. 470p.

SCOGNAMILLO-SZABÓ, M.V.R. et al. Involvement of corticoid and cytokines in the anti-inflammatory effect of acupuncture on carrageenan-induced peritonits in rats. Journal of Chinese Society of Traditional Veterinary Science, v.3, p.86-95, 2004.

SHEN, X. et al. Effect of combined laser acupuncture on knee osteoarthritis: a pilot study. Lasers Medicine Science. Disponível em: <http://www.springerlink.com/content/p184h8787t7wl15g/>. doi 10.1007/s10103-007-0536-9.

ULETT, G.A. Scientific studies and theories of acupuncture action. In: ZIMMERMANN, M. Beyond yin and yang: how acupuncture really works. St. Louis: Warren H. Green, 1992. p.38-54.

ULETT, G.A. et al. Electroacupuncture: Mechanisms and clinical application. Biological Psychiatry, v.44, p.129-138, 1998.

UM, S.W.; et al. Thermographic evaluation for the efficacy of acupuncture on induced chronic arthritis in the dog. Journal of Veterinary Medical Science, v.67, n.12, p.1283-1284, 2005.

USICHENKO T.I. et al. Auricular acupuncture for postoperative pain control: a systematic review of randomised clinical trials. Anaesthesia. v.63, n.12, p.1343-1348, 2008

WANG, C. et al. Electroacupuncture (EA) modulates the expression of NMDA receptors in primary sensory neurons in relation to hyperalgesia in rats. Brain Research, v.1120, p.46-53, 2006.

WONG, R.H.L. et al. Analgesic effect of electroacupuncture in postthoracotomy pain: a prospective randomized trial. Annals of Thoracic Surgery, v.81, p.2031-2036, 2006.

XIE, H.; PREAST, V. Xie's veterinary acupuncture. Oxford: Blackwell, 2007. 376p.

YANG, J. et al. Effect of hypothalamic supraoptic nucleus on acupuncture analgesia in the rat. Brain Research Bulletin, v.75, p.681-686, 2008.

ZETUN, M.C. et al. Eletroestimulação dos acupontos Zulinqi (vb41) e Waiguan (ta5) sobre parâmetros cardiorrespiratórios e consumo do anestésico isofluorano em gatas submetidas à ovariosalpingohisterectomia eletiva. In: CONGRESSO BRASILEIRO DE CIRURGIA E ANESTESIOLOGIA VETERINÁRIA, 8., 2008, Recife, PB. Anais... Recife: CBCAV, 2008. CD Room.

ZHANG, R.X. et al. Involvement of opioid receptors in electroacupuncture-produced anti-hyperalgesia in rats with peripheral inflammation. Brain Research, v.1020, p.12-17, 2004.

ZHANG, R.X. et al. Electroacupuncture attenuates bone cancer pain and inhibits spinal interleukin- $1 \beta$ expression in a rat model. Anesthesia and Analgesia, v.105, p.1482-1488, 2007.

ZHANG, R.X. et al. Mu opioid receptor-containing neurons mediate electroacupuncture-produced anti-hyperalgesia in rats with hind paw inflammation. Brain Research, v.1048, p.235$240,2005$. 